\title{
The development of a highly sensitive urea sensor due to the formation of an inclusion complex between urea and sulfonated- $\beta$-cyclodextrin
}

\author{
Anita Hamilton ${ }^{*}, 1$, Carmel B. Breslin ${ }^{1}$ \\ Department of Chemistry, National University of Ireland Maynooth, Maynooth, Co. Kildare, Ireland
}

\section{A R T I C L E I N F O}

\section{Article history:}

Received 14 September 2013

Received in revised form

24 December 2013

Accepted 15 January 2014

Available online 4 February 2014

\section{Keywords:}

Polypyrrole

Urea sensor

Urease

Cyclodextrin

Inclusion complexation

\begin{abstract}
A B S T R A C T
A highly sensitive urea sensor was developed by incorporating the urease enzyme (Urs) into a polypyrrole film (PPy) in one simple electropolymerisation step, using a sulfonated- $\beta$-cyclodextrin dopant. This PPyUrs-SCD film has a superior sensitivity of $5.79 \mu \mathrm{C} \mu \mathrm{M}^{-1}$ and detection in the region of $1.0 \times 10^{-10} \mathrm{M}$ urea, which is greater than other urea sensors reported in the literature. This is due to the formation of an inclusion complex between urea and a sulfonated- $\beta$-cyclodextrin host in an aqueous solution, which was established using electrochemical techniques. Cyclic voltammetry was used to investigate the effect of an excess concentration of the sulfonated- $\beta$-cyclodextrin on the currents recorded for urea. A clear reduction in the current was observed upon the addition of the sulfonated- $\beta$-cyclodextrin. The formation constant, $K_{f}$, was computed as $2745 \pm 300 \mathrm{M}^{-1}$, indicating the formation of a relatively strong inclusion complex. In addition, a 1:1 stoichiometry for the inclusion complex was deduced from a Job's plot analysis.
\end{abstract}

(C) 2014 Elsevier Ltd. All rights reserved.

\section{Introduction}

The simplest method of monitoring urea concentration is to immobilise the urease enzyme (Urs) onto an electrode. This has been widely investigated throughout the literature and proves to be the most promising approach [1]. The urease enzyme can be immobilised onto an electrode by covalent binding to a conducting polymer film, or by entrapment during the electrodeposition of the polymer film onto the electrode. A wide range of conducting polymers has been used for the entrapment of urease, including polypyrrole (PPy), polyaniline (PAni) and polythiophene, and their derivatives [2]. Additionally, a large number of dopant anions have been incorporated into the polymer film during electropolymerisation. The chosen dopant anion is important in the growth of polypyrrole films as different sized ions lead to different dopant levels within the polypyrrole film $[3,4]$ and dopants range in size from simple chloride ions to polyanions such as polyacrylate, polystyrene sulfonate and sulfonated-cyclodextrins.

Cyclodextrins (CD) are a series of naturally occurring macrocyclic oligosaccharides formed from $\alpha-1,4$-linked-D-glucopyranose

\footnotetext{
* Corresponding author. Tel.: +353 85 1556525; fax: +35317083815

E-mail addresses: anita.hamilton@nuim.ie (A. Hamilton),

carmel.breslin@nuim.ie (C.B. Breslin).

1 ISE member.
}

units [5-7]. The primary and secondary hydroxyl groups on the exterior of the cyclodextrin are polar, while the hydrogens inside of the cyclodextrin are apolar. As a result, most cyclodextrins are soluble in water with a hydrophilic exterior and a hydrophobic interior cavity [8]. This structural property of cyclodextrins gives rise to their complexation ability in aqueous media and inclusion complexes are formed with appropriately sized guests through non-covalent interactions, such as, hydrogen bonding, hydrophobic interactions and electrostatic interactions.

Cyclodextrins can be chemically modified by replacing the hydroxyl groups on both the primary and secondary rims with a variety of appropriate alkyl or sulfate groups in order to enhance the solubility of the cyclodextrin. In addition, substituting the hydroxyl groups on the cyclodextrin can either improve or inhibit the binding affinity of the cyclodextrin [9]. Negatively charged cyclodextrins can be obtained by the substitution of the hydroxyl groups with sulfonate groups.

This paper is focused on the development of a novel urea sensor formed by the entrapment of the urease enzyme within a polypyrrole matrix doped with sulfonated- $\beta$-cyclodextrin (SCD). In addition, we report on the formation of an inclusion complex between the SCD acting as a host molecule and urea as the guest molecule. Urea was chosen as it is an important compound in both the environmental and medical industries and to be able to monitor changes in urea concentration is vital. 


\section{Experimental}

\subsection{Chemicals}

The chemicals used throughout this study were purchased from Sigma-Aldrich or its subsidiary company Fluka. All chemicals were used as supplied expect for pyrrole which was vacuum-distilled and stored in the dark at $-20^{\circ} \mathrm{C}$ prior to use. All other solutions were made from a stock solution of $\mathrm{pH}$ 7.0, 0.05 $\mathrm{M}$ phosphate buffer, which was initially prepared using distilled water. This concentration of phosphate buffer was chosen as higher concentrations are known to interfere with the biocatalytic activity of urease, whereas lower concentrations have insufficient conductivity [10]. For the complexation study, $0.30 \mathrm{M} \mathrm{NaCl}$ was added to the $\mathrm{pH} 7.0,0.05 \mathrm{M}$ phosphate buffer in order to raise the conductivity of the solution, as the SCD has a very high conductivity of $21.40 \mathrm{mS}$, at room temperature, and an ionic strength of $2.25 \mathrm{M}$ for a $0.05 \mathrm{M}$ concentration. All of the solutions were freshly prepared before each experiment.

\subsection{Instrumentation}

Potentiostatic and cyclic voltammetry experiments were carried out using a Solartron Potentiostat Model 1287. All measurements were performed at room temperature (approximately $25^{\circ} \mathrm{C}$ ) in a standard three-electrode cell with a platinum (Pt) or glassy carbon (GC) working electrode, a high surface area platinum wire counter electrode and a SCE reference electrode. The Pt and GC electrodes ( $4 \mathrm{~mm}$ in diameter) were encased in a larger Teflon ${ }^{\circledR}$ sheath and set in place using a non-conducting epoxy resin. The electrical contact was made with a copper wire attached using a highly conducting silver-loaded resin. The working electrodes were polished to a mirror finish using 30,15, 6 and $1 \mu \mathrm{m}$ diamond suspensions on microcloth (Buehler), sonicated in distilled water and then in ethanol to remove any polishing residues, and finally rinsed with distilled water and dried.

\subsection{Fabrication of the Urs immobilised into polypyrrole (PPy) films}

The urease enzyme, (Urs) was immobilised into the polypyrrole (PPy) films in a single-step process by physical entrapment of the enzyme into the conducting polymer during electrodeposition. The films were electrochemically prepared at a fixed potential of $0.70 \mathrm{~V}$ vs. SCE from an aqueous solution containing the pyrrole monomer $(0.50 \mathrm{M})$, Urs $\left(4000 \mathrm{mg} \mathrm{L}^{-1}\right)$ and sulfonated$\beta$-cyclodextrin $(0.02 \mathrm{M})$ to form the PPy-Urs-SCD films. These parameters were chosen as lower concentrations of pyrrole monomer lead to insufficient polymer growth in the presence of the urease enzyme, the applied potential of $0.70 \mathrm{~V} \mathrm{vs}$. SCE provides a uniform polymer layer with little defects, unlike those formed at higher potentials and the concentration of SCD was fixed at $0.02 \mathrm{M}$. Although the anionic CD may give rise to an increase in the viscosity of the solution, there is little to no increase in the solution viscosity at this concentration $[11,12]$. The polymer films were deposited until a fixed charge of $0.10 \mathrm{C} \mathrm{cm}^{-2}$ was achieved. The thickness of the films obtained was approximated as $3.55 \mu \mathrm{m}$, which was theoretically calculated using the charge thickness ratio derived by Diaz et al. [13] for a simple chloride dopant. In this analysis it is assumed that $1.0 \mathrm{C} \mathrm{cm}^{-2}$ of charge passed is equivalent to $2.5 \mu \mathrm{m}$ of polymer film. It is important to mention that the theoretical values of thickness obtained for the PPy-Urs-SCD films are only an approximation, as the films doped with the large anionic groups may not have the same charge to polymer thickness ratio as the PPy films doped with simple chloride anions [14,15]. These films were characterised using SEM and EDX analysis and then investigated as suitable sensors for the detection of urea. Calibration

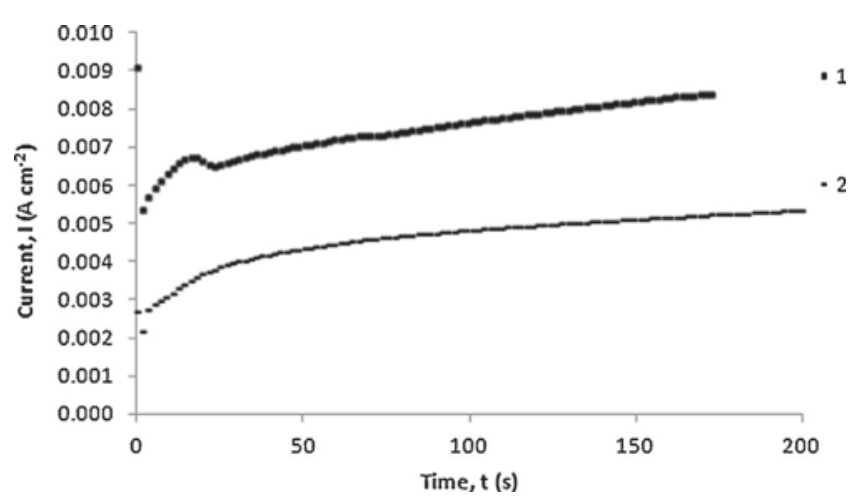

Fig. 1. Current plotted as a function of time for the formation of $\mathbf{m} 1$ PPy-Urs-SCD and -2 PPy-Urs-Cl on a Pt electrode at 0.70 V vs. SCE.

curves were generated from the average of six experiments $(n=6)$ and the uncertainties of both the sensitivity and detection limit were calculated from Equation 1.

Standard Error $=\frac{\text { Standard Deviation } / \sqrt{n}}{\text { Mean Current of } n} \times \frac{100}{1}$

\subsection{Investigation of the formation of an inclusion complex between urea and $S C D$}

The cyclic voltammogram experiments were recorded at different scan rates, ranging from 300 to $5 \mathrm{mV} \mathrm{s}^{-1}$ in the potential interval of -0.60 to $0.80 \mathrm{~V}$ vs. SCE. The urea concentration was maintained fixed at $5.0 \times 10^{-4} \mathrm{M}$ in the supporting electrolyte, while the concentration of the sulfonated- $\beta$-cyclodextrin was varied to give solutions with an excess of the SCD. Jobs plots were constructed using the voltammetry approach by recording the current measured at a fixed potential in solutions with different mole fractions of the sulfonated- $\beta$-cyclodextrin, SCD, and the urea. Each experiment was performed a minimum of six times $(n=6)$ and the average was obtained. It is this average that is presented and discussed.

\section{Results and Discussion}

\subsection{Formation of PPy-Urs-Cl and PPy-Urs-SCD using a potentiostatic mode}

For the purpose of this study, two different PPy-Urs polymer films were generated, using either a simple chloride anion or the sulfonated- $\beta$-cyclodextrin (SCD) as the dopant species. In both cases, the urease enzyme was incorporated into the polymer film via physical entrapment during the deposition of the polymer, by applying a fixed potential of $0.70 \mathrm{~V}$ vs. SCE to the monomer containing solution. The polypyrrole films were deposited from a $0.10 \mathrm{M} \mathrm{NaCl}$ solution in the presence and absence of urease to give PPy-Urs-Cl and PPy-Cl polymer films. Similarly, PPy-Urs-SCD and PPy-SCD films were formed using the sulfonated- $\beta$-cyclodextrin (SCD), which is a polyanion with a high conductivity, as the dopant species.

The current-time plots for the chloride-containing films differ greatly from those of the SCD-containing films, as observed in Fig. 1. Initially, there is a rapid decrease in the current, which arises from the charging of the double layer. This is then followed by a fast rise in the current, which corresponds to the nucleation and growth of the polymer film [16]. Then, for the chloride-containing films, there is a further more gradual increase in the current as the polymer is deposited onto the working electrode to give a higher surface area. However, with the SCD-containing films, this rapid increase in current reaches a maximum value within a number of seconds, typically $20 \mathrm{~s}$, which is characteristic of the SCD electrolyte [17], at 
(a)

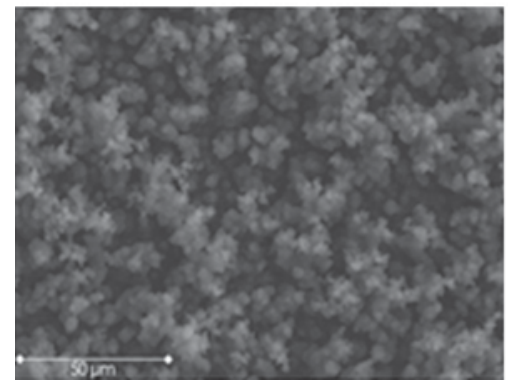

(b)

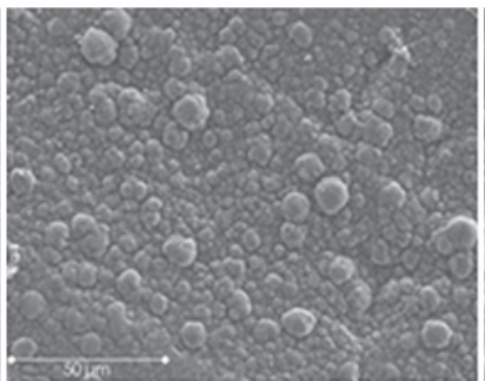

(c)

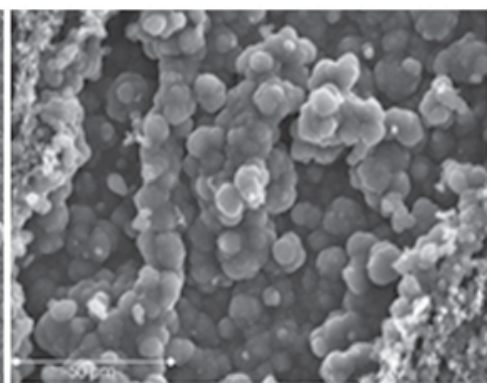

Fig. 2. Scanning electron micrograph of (a) PPy-Urs-Cl, (b) PPy-SCD and (c) PPy-Urs-SCD electrodeposited on a Pt electrode at $0.70 \mathrm{~V}$ vs. SCE to a charge of $10.48 \mathrm{C}$ cm ${ }^{-2}$.

which time the current begins to decrease again. This is followed by a further more gradual increase in the current as the polymer becomes deposited onto the working electrode $[14,18]$.

The unusual shape of the current-time transients may be due to the polyelectrolyte properties of the SCD [9]. As no other supporting electrolyte was used, these polyanions will migrate to the positively charged surface of the working electrode on the application of the potential. This gives rise to a high local concentration of the SCD anions during the initial stages of electropolymerisation, which allows the electropolymerisation reaction to proceed at a very high rate once the monomer oxidation is initiated. However, as the electropolymerisation reaction proceeds, the concentration of the SCD anions is reduced as they are doped within the polypyrrole layers deposited onto the electrode, and the rate of the electropolymerisation reaction is now dominated by the transport and diffusion of the large SCD anions to the interface. The diffusion of the SCD anions is slow due to the size of the SCD with 7-11 sulfonate groups and this gives rise to a drop in the rate of electropolymerisation which is consistent with the slight dip in the current at approximately 20 s, Fig. 1.

\subsection{Characterisation of the polymer films using SEM and EDX}

The surface morphologies of the PPy-Urs-Cl, PPy-SCD and PPyUrs-SCD polymer films were characterised by scanning electron microscopy. Fig. 2 shows that the urease containing polymer films have a fibrous morphology, due to the incorporated enzyme, whereas the films without urease are very different and do not show any evidence of this fibrous morphology. In addition, the PPy-SCD film (Fig. 2b) has the typical cauliflower morphology of polypyrrole owing to the nuclei forming quickly in the presence of the doping anions and the bulk polymer subsequently growing

(a)

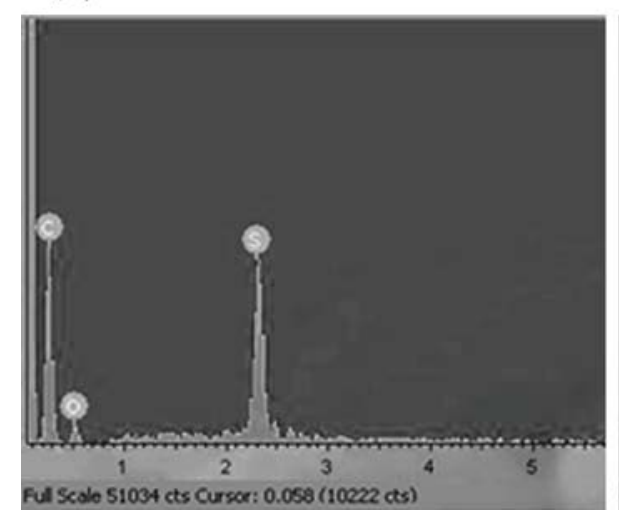

preferentially around the nucleation sites [19]. Although SEM analysis cannot give concrete evidence of the roughness of a sample, it is apparent from the scanning electron micrographs that the PPyUrs-SCD film (Fig. 2c) is more porous. This is most likely due to the incorporation of both the SCD and the urease enzyme within the polypyrrole matrix. Both species are relatively large, giving rise to a more porous surface morphology.

EDX measurements were carried out on the PPy-SCD and PPyUrs-SCD polymer films in an attempt to detect the urease enzyme and typical spectra are shown in Fig. 3. The EDX spectra clearly show the presence of sulfur, arising from the sulfonated groups on the sulfonated- $\beta$-cyclodextrin. The dopant anion is incorporated during the electropolymerisation of pyrrole at $0.70 \mathrm{~V}$ vs. SCE, to balance the positive charge on the oxidised PPy. The dopant anion is important in the growth of polypyrrole films as different sized ions lead to different dopant levels within the polypyrrole film $[3,4]$. The significant difference between the EDX spectra in the presence and absence of urease is the presence of the nickel in the PPy-Urs-SCD film, which is absent in the PPy-SCD film. This nickel is contained in the active site of the Jack Bean urease enzyme [1], and its presence in the EDX spectrum of PPy-Urs-SCD is clear evidence that the urease is incorporated successfully into the polymer film.

\subsection{Sensing studies of the polymer films}

After the preparation and rinsing of the modified electrode, the electrode was then cycled in a pH 7.0, $0.05 \mathrm{M}$ phosphate buffer solution between -0.60 and $0.80 \mathrm{~V}$ vs. SCE until a steady state was achieved. The modified electrode was then removed, rinsed and placed into a low concentration of urea in the phosphate buffer solution and cycled for ten cycles, then rinsed again and placed into a solution with a slightly higher concentration of urea. This (b)

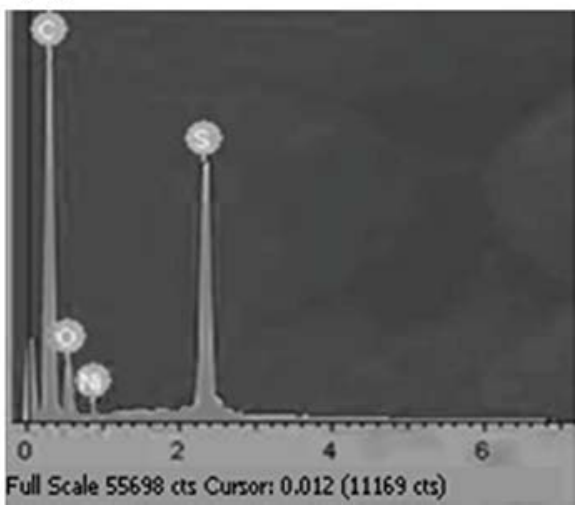

Fig. 3. EDX analysis of (a) PPy-SCD and (b) PPy-Urs-SCD electrodeposited onto a platinum electrode at an applied potential of $0.70 \mathrm{~V}$ vs. SCE until a charge of $10.48 \mathrm{C} \mathrm{cm}^{-2}$ was reached. 


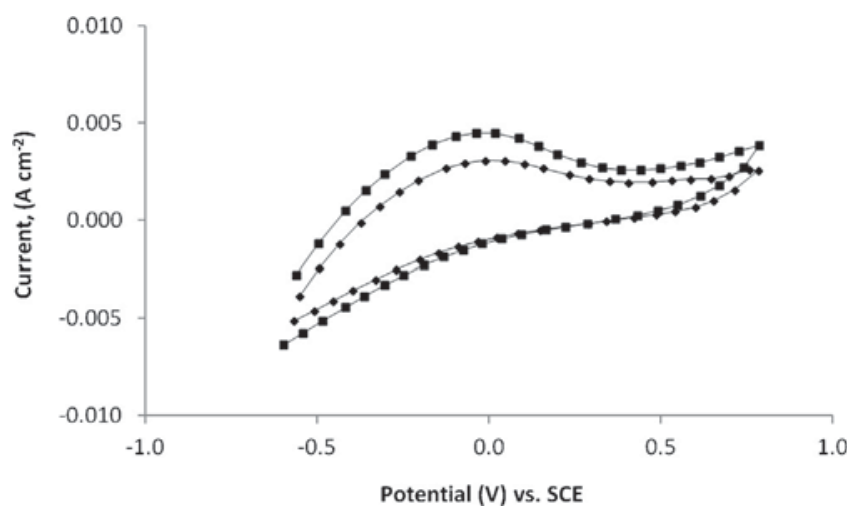

Fig. 4. Cyclic voltammograms recorded for the PPy-Urs-Cl polymer film in the presence and $\downarrow \Delta$ absence of urea, similar CVs were recorded for the other polymer films.

was repeated over a large concentration range, with rinsing of the modified electrode carried out between each solution in order to avoid transfer and contamination of the solutions.

Fig. 4 shows the cyclic voltammograms recorded for the modified electrode in the absence and presence of $1.0 \times 10^{-4} \mathrm{M}$ urea. It is evident that there is an increase in the current in the presence of urea but this extends over the entire electrochemical window, with no well-defined peak in the current. Although most sensors are amperometric [20], where the current at a fixed potential is monitored, a different approach was taken in this study. The entire potential range ( -0.60 to $0.80 \mathrm{~V}$ vs. SCE) was used and the charge over this potential window was computed, producing a coulombometric sensor. As the presence of urea leads to an increase in the charge, Fig. 4, the charge arising in the absence of urea, from the background phosphate buffer, can be easily subtracted. Hence, all the results presented are given with the background charge subtracted, and represent the true charge arising from the urea.

Typical calibration curves recorded for the PPy-Cl, PPy-Urs-Cl, PPy-SCD and PPy-Urs-SCD films are presented in Fig. 5 with the charge plotted as a function of the urea concentration. The sensitivity of the films towards urea at the higher concentrations, i.e. the linear regions obtained from $0.003 \mathrm{M}$ to $0.01 \mathrm{M}$, was calculated at $0.43,0.76,2.29$ and $5.79 \mu \mathrm{C} \mu \mathrm{M}^{-1}$ for the PPy-Cl, PPy-Urs-Cl, PPy-SCD and PPy-Urs-SCD films, respectively, with detection in the region of $1.0 \times 10^{-10} \mathrm{M}$ for the PPy-SCD and PPy-Urs-SCD films.

Surprisingly, the PPy-SCD polymer film has a greater sensitivity towards urea than the PPy-Urs-Cl film, which only has a sensitivity of $0.76 \mu \mathrm{C} \mu \mathrm{M}^{-1}$ compared to $2.29 \mu \mathrm{C} \mu \mathrm{M}^{-1}$ for the SCD-containing film. However, the presence of the urease greatly enhances the sensitivity of the polymer film due to its catalytic activity on the urea [10]. This is seen on a comparison of the sensing performance of PPy-Cl and PPy-Urs-Cl and it is even more evident on comparing the PPy-Urs-SCD and PPy-SCD films, Fig. 5(a). The PPy-Urs-SCD film

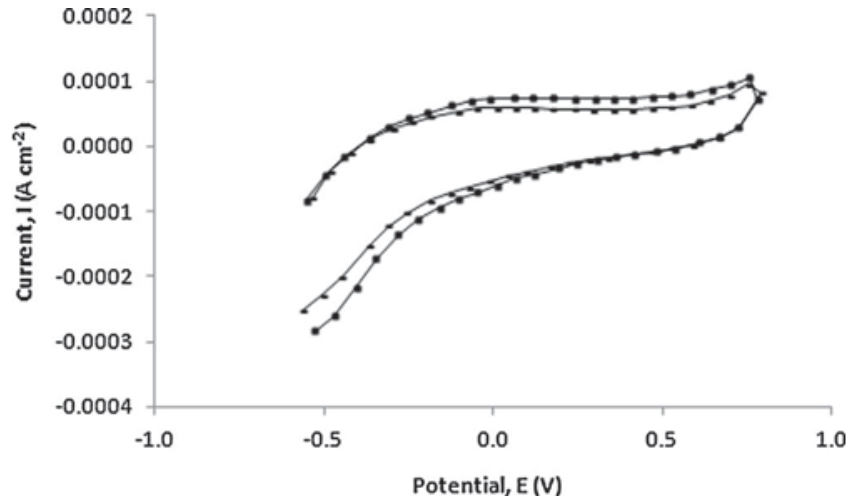

Fig. 6. Cyclic voltammograms obtained by cycling a bare glassy carbon electrode in $-\mathrm{pH} 7.0$ phosphate buffer solution and $1.0 \times 10^{-4} \mathrm{M}$ urea made in a $\mathrm{pH} 7.0$ phosphate buffer solution.

has superior sensitivity over the other films studied, Fig. 5(b), and has better detection limits than other sensors reported in the literature, which range from $1.0 \times 10^{-5}$ to $3.0 \times 10^{-1} \mathrm{M}$ urea $[21,22]$. This is highlighted in Table 1, where the linear range, detection limit and response times are compared for different sensors. This indicates that both the urease and the SCD dopant anion have important roles to play in the sensitivity of these films for urea detection.

\subsection{Investigation of the formation of an inclusion complex} between urea and SCD

It is well known that cyclodextrins can form inclusion complexes with a variety of guest molecules and the formation of an inclusion complex between the SCD and the urea is consistent with the enhanced sensitivity of the SCD-containing films. The urea is neutral and should not be repelled by the negatively charged SCD. Furthermore, the urea is sufficiently small to fit inside the cavity of the SCD. Cyclic voltammetry was employed to investigate these interactions as it is commonly employed to study the interactions between host and guest species $[23,24]$.

The cyclic voltammograms recorded in the phosphate buffer solution in the absence and presence of $1.0 \times 10^{-4} \mathrm{M}$ urea at the bare glassy carbon electrode are shown in Fig. 6. It is clearly evident that no significant oxidation or reduction peaks are observed over this potential region [25]. However, an increase in current is observed over the entire potential range and therefore, although the peak oxidation or peak reduction currents cannot be plotted, the currents at a fixed potential may be recorded for the anodic and cathodic processes.

In order to investigate the formation of an inclusion complex between urea and the SCD, cyclic voltammograms were recorded at varying scan rates for $5.0 \times 10^{-4} \mathrm{M}$ urea in the absence and presence of $2.0 \times 10^{-2} \mathrm{M} \mathrm{SCD}$ in the buffer solution. The currents recorded at (a)

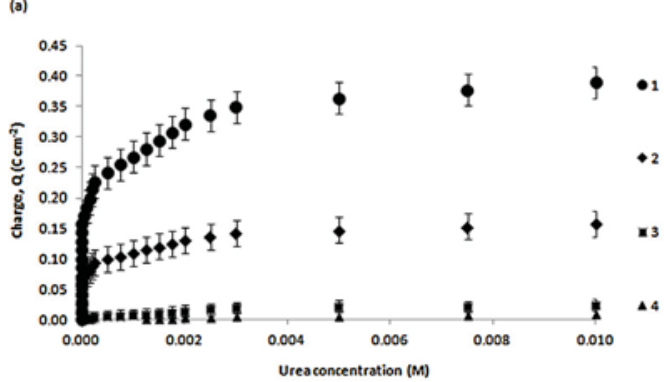

(b)

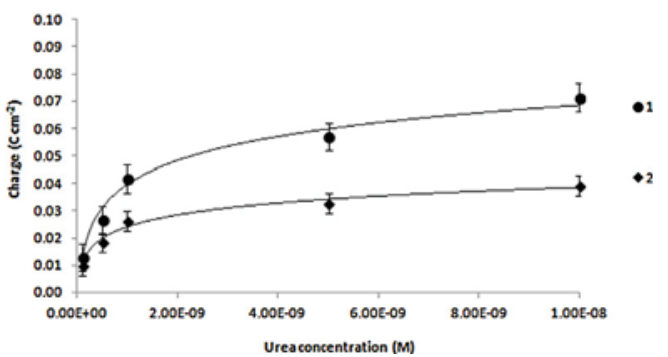

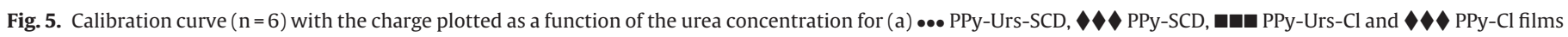
and (b) $\bullet \bullet$ PPy-Urs-SCD and $\bullet$ PPy-SCD. 
Table 1

Comparison of characteristics of urea biosensors (taken from [20,21]).

\begin{tabular}{|c|c|c|c|c|c|}
\hline Matrix used & Transducer & Stability & Linear range & Detection limit & Response time \\
\hline Polypyrrole-urease-SCD* & Coloumbometric & - & & & \\
\hline Polypyrrole & Potentiometric & - & $31.8 \mathrm{mV} / \mathrm{dl}$ & - & - \\
\hline Polypyrrole & Amperometric & 2 weeks & - & 60 ug/l & - \\
\hline Poly pyrrole & Amperometric & - & - & $3 \mathrm{ppm}$ & - \\
\hline $\begin{array}{l}\text { Poly }(N \text {-3-aminopropylpyrrole- } \\
\text { co-pyrrole) } \\
\text { film }\end{array}$ & Potentiometric & 2 months & $27.5 \mathrm{mV} / \mathrm{dl}$ & & $25-50 \mathrm{~s}$ \\
\hline Urease onto $\mathrm{Si} / \mathrm{SiO} 2$ Structure & $\mathrm{CV}$ measurements & Few days & $22 \mathrm{mV} / \mathrm{P}$ urea & $1 \mathrm{mM}$ & - \\
\hline $\begin{array}{l}\text { Polypyrrole and polyion } \\
\text { complex }\end{array}$ & Potentiometric & $\begin{array}{l}\text { Operational } \\
\text { stability of }>10 \\
\text { usages } / 0.1 \mathrm{M} \\
\text { Tris- } \mathrm{HCl}\end{array}$ & $3 \times 10^{-3}$ to $3 \times 10^{-1} \mathrm{M}$ & $3 \times 10^{-5} \mathrm{M}$ & $20 \mathrm{~s}$ \\
\hline $\begin{array}{l}\text { Polyurethane acylate } \\
\text { polymeric membrane }\end{array}$ & Potentiometric (ISFET) & $>30$ days $/ 4 \circ \mathrm{C}$ & $0.04-36 \mathrm{mM}$ & $0.04 \mathrm{mM}$ & $30 \mathrm{~s}$ to $5 \mathrm{~min}$ \\
\hline $\begin{array}{l}\text { Poly( } N \text {-vinyl carbazole/stearic } \\
\text { acid) Langmuir-Blodgett film }\end{array}$ & Potentiometric & 5 weeks at $4 \circ \mathrm{C}$ & $0.5-68 \mathrm{mM}$ & $0.5 \mathrm{mM}$ & $2 \mathrm{~min}$ \\
\hline Triacetyl cellulose membrane & Optical & $\begin{array}{l}60 \text { days stored } \\
\text { wet } / 4 \circ C \text { (with } 20 \% \\
\text { loss) }\end{array}$ & $1-500 \mathrm{mM}$ & $1 \mathrm{mM}$ & $1-5 \min$ \\
\hline Polyurethane film & Optical & - & $0.7-8 \mathrm{mM}$ & $20 \mu \mathrm{M}$ & $20 \mathrm{~s}$ \\
\hline Nylon net & Amperometric & 4 days & $10^{-5}$ to $3 \times 10^{-4} \mathrm{M}$ & $10^{-5} \mathrm{M}$ & - \\
\hline
\end{tabular}

*The PPy-Urs-SCD film as described by authors.

$0.30 \mathrm{~V}$ vs. SCE were plotted as a function of the square root of the scan rate, and the resulting plots are presented in Fig. 7 showing that the currents are directly proportional to the square root of the scan rate, which proves that the reaction is diffusion controlled. This was also confirmed by plotting the logarithm of the current as a function of the logarithm of the scan rate, which yielded slopes near to 0.50 , which also show that the reaction is diffusion controlled. This is significant as it has been reported in the literature that voltammetric methods, such as cyclic voltammetry and rotating disc voltammetry, are only suitable in the analysis of inclusion complexes if the guest is under diffusion control [26]. Consequently, this electrochemical approach is ideal for probing the complexation between urea and the SCD [27].

The plots, presented in Fig. 7, show very good linearity with correlation coefficients of 0.988 and 0.970 for urea in the absence and presence of SCD, respectively, for the anodic currents. The slopes of the linear plots are very different in the presence and absence of the SCD. Although the anionic cyclodextrin may give rise to an increase in the viscosity of the solution, according to the literature, no significant change in the cyclodextrin viscosity is detected for concentrations up to $1.0 \times 10^{-2} \mathrm{M} \mathrm{SCD}[11,12]$. Therefore, these data are consistent with a decrease in the diffusion coefficient of urea in the presence of the SCD. The diffusion coefficients of urea were calculated from the slopes of these plots as $2.72 \times 10^{-6}$ and

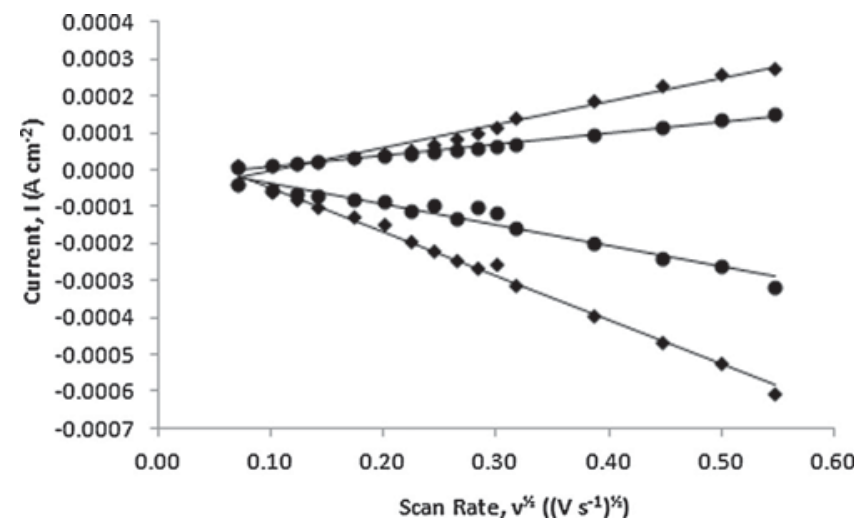

Fig. 7. Plot of the current, $I$, recorded at $0.30 \mathrm{~V}$ vs. SCE as a function of $\mathrm{v}^{1 / 2}$ on a GC disc electrode in a NaCl-phosphate buffer solution, pH 7.0, for $5.0 \times 10^{-4} \mathrm{M}$ urea in the $\downarrow$ absence and $\bullet \bullet$ presence of $2.0 \times 10^{-2} \mathrm{M} \mathrm{SCD}$.
$6.30 \times 10^{-7} \mathrm{~cm}^{2} \mathrm{~s}^{-1}$ using an $n$ value of 2 [28] in the absence and presence of SCD, respectively, using the well-known RandlesSevcik equation, Equation 2.

$I=k n^{3 / 2} D^{1 / 2} c v^{1 / 2}$

These are in reasonably good agreement with the diffusion coefficient values of $3.70 \times 10^{-6}$ to $8.30 \times 10^{-6} \mathrm{~cm}^{2} \mathrm{~s}^{-1}$ reported in the literature [29-31]. This decrease in the diffusion coefficient can be attributed to the formation of an inclusion complex between urea and the SCE. The sulfonated cyclodextrin is very large and bulky compared to urea and this will influence the diffusion of urea when urea is confined within the cavity of the cyclodextrin. Indeed, it has been shown that the diffucion coefficient of a guest molecule is reduced when included inside the cavity of a cyclodextrin [11].

\subsection{Stoichiometry of an inclusion complex between urea and SCD}

The stoichiometry of the urea and the SCD complex was investigated using the Job's plot or continuous variation method [32-34]. To carry this out, $0.01 \mathrm{M}$ stock solutions of urea and SCD were made up in the $\mathrm{NaCl}$-phosphate buffer and mixed together in different ratios in order to keep the total concentration constant while varying the mole fractions of urea from 0.0 to 1.0 in increments of 0.1 . The volumes of each stock solution and mole fractions of urea employed for the Job's method are given in Table 2. Cyclic voltammograms were recorded in each of these solutions and,

Table 2

Volumes of the SCD and urea stock solutions and mole fractions of urea used for the Job's plot 422 measurements, where the total volume is $10.0 \mathrm{~mL}$.

\begin{tabular}{lccl}
\hline Solution number & $\begin{array}{l}\text { Volume of SCD } \\
(\mathrm{mL})\end{array}$ & $\begin{array}{l}\text { Volume of urea } \\
(\mathrm{mL})\end{array}$ & $\begin{array}{l}\text { Mole fraction } \\
\text { of urea }\end{array}$ \\
\hline 1 & 10.0 & 0.0 & 0.0 \\
2 & 9.0 & 1.0 & 0.1 \\
3 & 8.0 & 2.0 & 0.2 \\
4 & 7.0 & 3.0 & 0.3 \\
5 & 6.0 & 4.0 & 0.4 \\
6 & 5.0 & 5.0 & 0.5 \\
7 & 4.0 & 6.0 & 0.6 \\
8 & 3.0 & 7.0 & 0.7 \\
9 & 2.0 & 8.0 & 0.8 \\
10 & 1.0 & 9.0 & 0.9 \\
11 & 0.0 & 10.0 & 1.0 \\
\hline
\end{tabular}




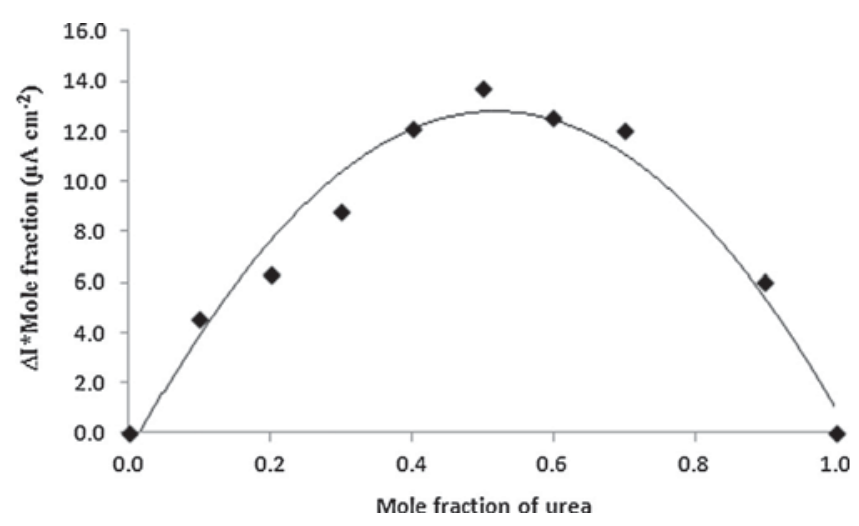

Fig. 8. Job's plot curve where the change in the currents recorded at $0.30 \mathrm{~V}$ vs. SCE are plotted as a function of the mole fraction of urea in a urea and SCD solution in a NaCl-phosphate buffer, at a $\mathrm{pH}$ of 7.0.

because the currents increase with increasing mole fractions of urea, a Job's plot was generated by following the changes in the currents recorded at a fixed potential, using the relationship given in Equation 3 [35].

$\Delta i=i_{0}-i_{x}$

Here $i_{0}$ and $i_{x}$ are the currents obtained at $0.30 \mathrm{~V}$ vs. SCE for urea in the absence and presence of SCD, respectively. These $\Delta i$ values were then multiplied by the corresponding mole fraction ( $\Delta i^{*}$ mole fraction) and the product was plotted as a function of the mole fraction of urea. A typical Job's plot is presented in Fig. 8. A clear maximum value is observed at a mole fraction of 0.50 , which confirms that the urea and SCD bind and form an inclusion complex in a 1:1 stoichiometric ratio [36], i.e., one urea molecule is included in a single SCD cavity.

\subsection{Determination of $K_{f}$ in the presence of the sulfonated- $\beta$-cyclodextrin}

In order to calculate the formation constant of the inclusion complex, an excess concentration of the SCD was added to the urea-containing solution to drive the equilibrium to favour the complexed urea-SCD species. Fig. 9 shows the cyclic voltammograms recorded for $1.0 \times 10^{-4} \mathrm{M}$ urea in the presence of increasing concentrations of SCD, up to a large excess of $2.0 \times 10^{-2} \mathrm{M}$. There is a considerable reduction in the current with increasing concentrations of the SCD, and this effect is more clearly shown in Fig. 10. These data indicate that the urea is more difficult to oxidise in the presence of the SCD, and again this points to the formation of an

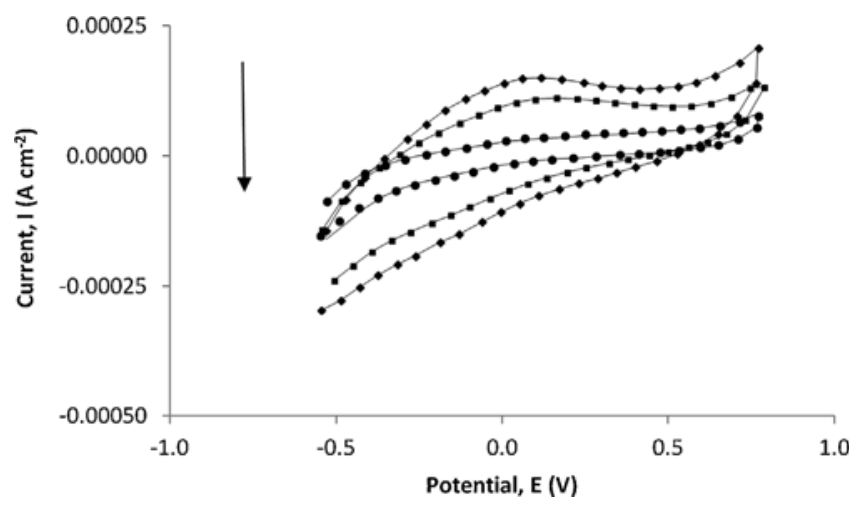

Fig. 9. Cyclic voltammograms recorded for $1.0 \times 10^{-4} \mathrm{M}$ urea in the absence and presence of increasing concentrations of SCD: the current decreases with increasing $\mathrm{SCD}$ concentration, i.e., from $0 \mathrm{M} / 1.0 \times 10^{-4} \mathrm{M}$ up to $2.0 \times 10^{-2} \mathrm{M}$.

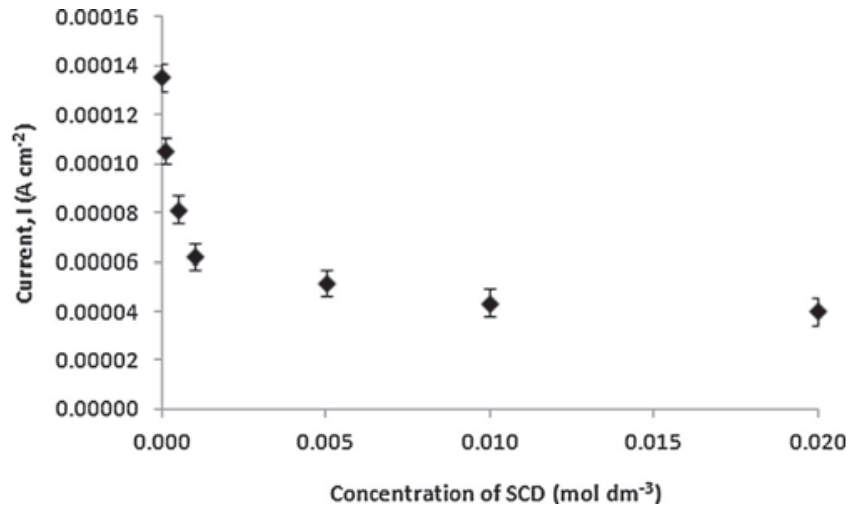

Fig. 10. Current for urea, recorded at $0.30 \mathrm{~V}$ vs. SCE, as a function of the SCD concentration $(n=6)$.

inclusion complex. In particular, the significant decrease in the recorded currents is consistent with the change in the diffusion coefficient of urea, where a lower diffusion coefficient is obtained when a guest in included within the host cavity $[11,37]$.

The current recorded at a fixed potential of $0.30 \mathrm{~V}$ vs. SCE is plotted as a function of the SCD concentration in Fig. 10. Again, there is a significant decay in the current as the concentration of the SCD is initially increased. Then the current reaches a near constant value when a large excess of the SCD is added to the solution. A similar trend was observed for the current recorded at potentials ranging from -0.25 to $0.80 \mathrm{~V}$ vs. SCE. Again this is consistent with the formation of an inclusion complex and indicates that the urea is included within the cavity when an excess of the SCD is present in the solution.

The $K_{f}$ value for the inclusion complex was calculated from the cyclic voltammetry data using Equation 4 [38].

$\frac{1}{[S G D]}=K_{f} \frac{(1-A)}{1-i / i_{0}}-K_{f}$

Here, $i_{0}$ represents the current obtained in the absence of the $\mathrm{SCD}, i$ represents the currents recorded in the presence of the SCD, [SCD] is the concentration of the $\mathrm{SCD}, A$ is a proportionality constant and $K_{\mathrm{f}}$ corresponds to the stability constant for the inclusion complex. A linear plot, with an $\mathrm{R}^{2}$ value of 0.986 , was obtained when the inverse of the SCD concentration was plotted as a function of $1 /\left(1-i / i_{0}\right)$, as shown in Fig. 11 . This linear relationship not only confirms the existence of a 1:1 inclusion complex but can also be used to calculate the stability constant $[6,38]$. Accordingly, the $K_{f}$ value for the inclusion complex was calculated as $2745 \pm 300 \mathrm{M}^{-1}$. This is quite high and indicates that a relatively strong inclusion complex

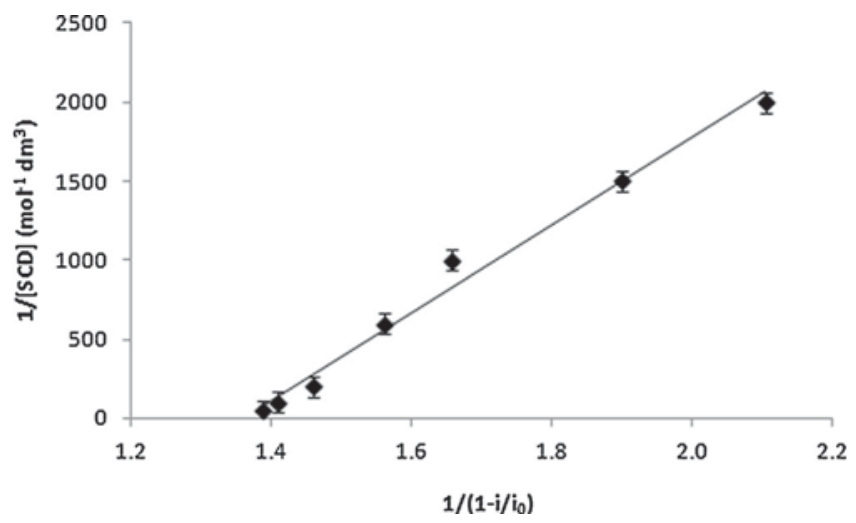

Fig. 11. Plot of $1 /[\mathrm{SCD}]$ as a function of $1 /\left(1-i / i_{0}\right),(n=6)$ for the evaluation of the stability constant, $K_{f}$, for urea in a NaCl-phosphate buffer solution at pH 7.0. 


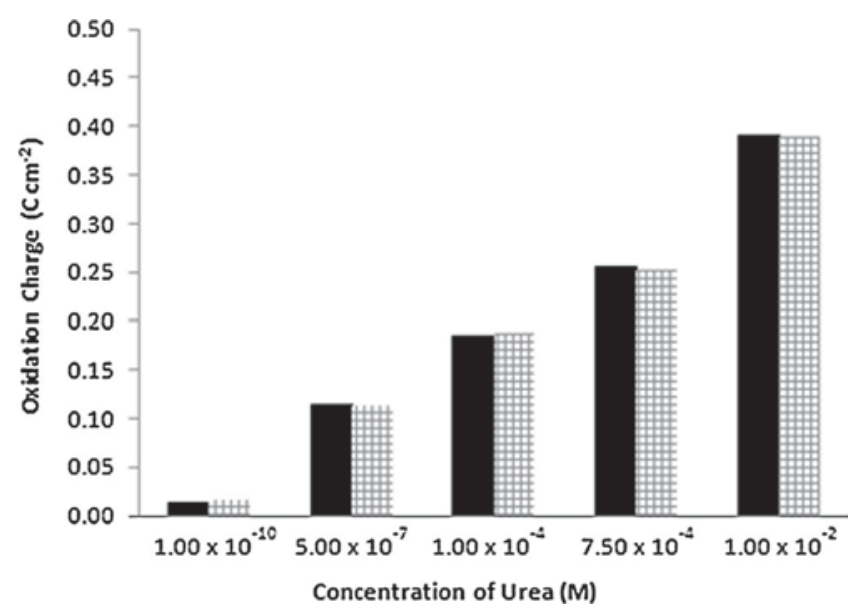

Fig. 12. Charge plotted at different urea concentrations in the - absence and presence of $1.0 \times 10^{-4} \mathrm{M}$ AA at the PPy-Urs-SCD polymer films.

is formed between the sulfonated- $\beta$-cyclodextrin and the urea as a guest molecule [11].

It is clear from the diffusion coefficients and the data presented in Figs. 7-11 that an inclusion complex is formed between urea and the SCD. This is consistent with the very good detection of urea shown in Fig. 5 for the PPy-Urs-SCD films. Furthermore, the greater sensitivity of the PPy-SCD compared to the PPy-Urs-Cl films can be explained in terms of this inclusion complex.

\subsection{Selectivity studies of the PPy-Urs-SCD polymer films}

The PPy-Urs-SCD film had excellent sensitivity in the electrochemical detection of urea and this polymer film was chosen and used in an attempt to eliminate the interference from ascorbic acid (AA). Because SCD is a large anionic species [39], this anionic character may be sufficient to repel the anionic ascorbate species [40]. Ascorbic acid has a pKa value of 4.10 and, at the biological $\mathrm{pH}$ of the phosphate buffer solution, dissociation of AA occurs to favour the ascorbate anion. The PPy-Urs-SCD polymer was deposited as detailed in Section 3.1 and cycled in a urea solution in the presence and absence of ascorbic acid. The urea concentration was varied from $1.0 \times 10^{-10}$ to $1.0 \times 10^{-2} \mathrm{M}$, while a fixed concentration of $1.0 \times 10^{-4} \mathrm{M} \mathrm{AA}$ was added to give AA/urea concentration ratios ranging from $1.0 \times 10^{6}$ to $1.0 \times 10^{-2}$. The oxidation charge was recorded in the urea solutions and then compared with the charge recorded in the mixed urea and AA solution. These data are summarised in Fig. 12. It is clear from these data that there is no interference observed when adding AA to the urea solution at the PPy-Urs-SCD polymer films. The oxidation charges obtained from cycling the polymer in a urea solution in the absence of AA are similar to those obtained on cycling the polymer film in a urea solution in the presence of AA. Regardless of the ratio of AA to urea, which is in the vicinity of $1.0 \times 10^{6}$ at the low concentrations of urea, there is no evidence of any interference from the added AA.

These data can be explained in terms of the negative charges of the sulfonated groups on the $\beta$-cyclodextrin within the PPyUrs-SCD film. Although the charge on the sulfonated groups may be balanced by an equal and opposite charge from the oxidised polypyrrole backbone $\left(\mathrm{PPy}^{+}\right)$, the- $\mathrm{SO}_{3}{ }^{-}$pendants will provide a highly negative local charge. In addition, some free- $\mathrm{SO}_{3}{ }^{-}$groups are likely to exist at the PPy-Urs-SCD surface [41]. It appears that the negatively charged sulfonated groups on the $\beta$-cyclodextrin are successful in repelling the anionic ascorbate from the surface of the electrode and hence, the urea can be detected without any interference from AA, as clearly shown in Fig. 12.

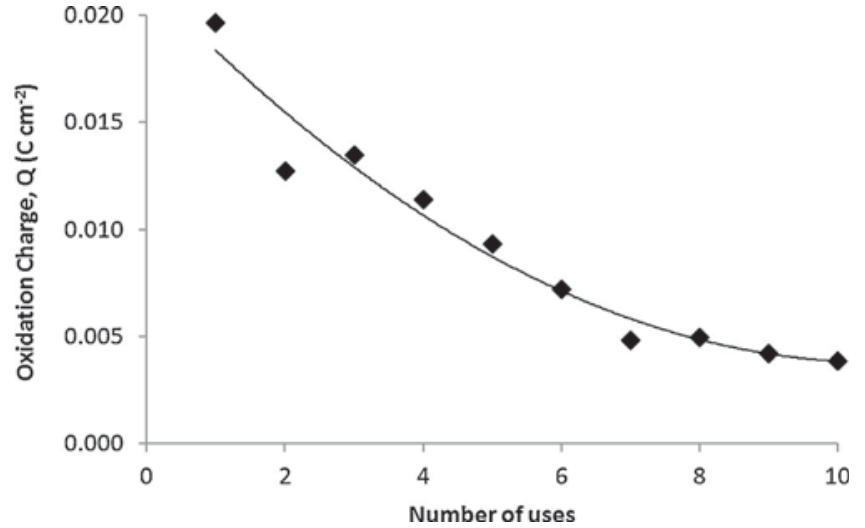

Fig. 13. Oxidation charge of the PPy-Urs-SCD film recorded in $0.003 \mathrm{~mol} \mathrm{dm}^{-3}$ urea plotted as a function of the number of uses.

\subsection{Stability studies of the PPy-Urs-SCD polymer films}

In order to explore and probe the stability of the PPy-Urs-SCD polymer films, parameters such as reusability and reproducibility of the polymer film were investigated. A cyclic voltammogram was obtained by cycling the polymer film initially in the background solution of $0.05 \mathrm{~mol} \mathrm{dm}^{-3}$ phosphate buffer solution, $\mathrm{pH} 7.0$, from -0.60 to $0.80 \mathrm{~V}$ vs. SCE, for 10 cycles. The polymer film was then transferred to a solution containing $0.003 \mathrm{~mol} \mathrm{dm}^{-3}$ solution of urea in $0.05 \mathrm{~mol} \mathrm{dm}^{-3}$ phosphate buffer at a $\mathrm{pH}$ of 7.0 , and cycled in the same window. The oxidation charge was recorded and the background subtracted to obtain the true oxidation charge. This process was repeated a total of ten times and the corresponding data are presented in Fig. 13, with the oxidation charge plotted as a function of the number of uses.

It is evident from Fig. 13 that there is a clear loss in the oxidation charge with repeated use. Indeed, there is a $25 \%$ loss in the charge from the first to the second use. This indicates that the PPy-UrsSCD polymer film is not suitable for re-use as the charge obtained decreases significantly with each use. This is comparable with studies done by Pandey, et al. [42] on other polymer-based urea sensors, such as polyaniline, and polypyrrole.

The reproducibility of the PPy-Urs-SCD polymer films in the detection of urea was investigated by electrodepositing a number of polymer films and cycling them initially in the background solution of $0.05 \mathrm{~mol} \mathrm{dm}^{-3}$ phosphate buffer solution, $\mathrm{pH} \mathrm{7.0,} \mathrm{and} \mathrm{then} \mathrm{in}$ a $0.003 \mathrm{~mol} \mathrm{dm}^{-3}$ urea in $0.05 \mathrm{~mol} \mathrm{dm}^{-3}$ phosphate buffer solution, $\mathrm{pH}$ 7.0. The true oxidation charge was computed and it is this charge that is presented in Fig. 14, where it is obvious that the oxidation

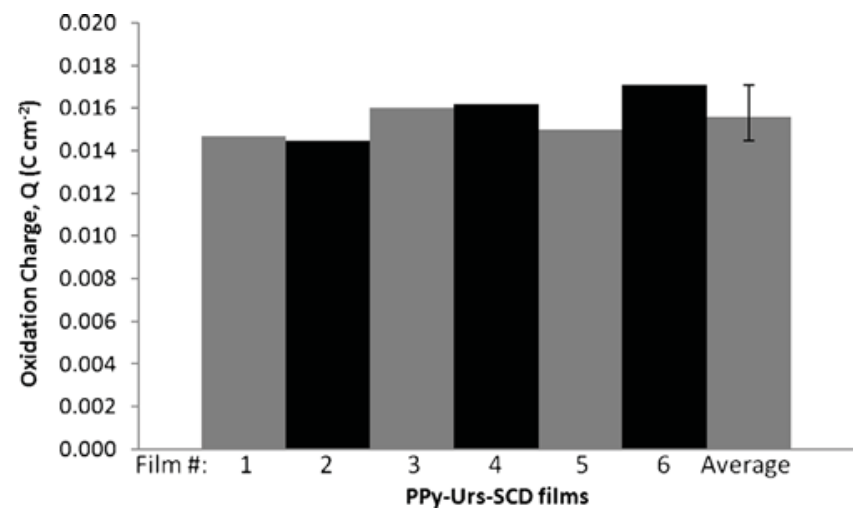

Fig. 14. Oxidation charges obtained for six different PPy-Urs-SCD polymer films by cycling in a $0.003 \mathrm{~mol} \mathrm{dm}^{-3}$ urea in $0.05 \mathrm{~mol} \mathrm{dm}^{-3}$ phosphate buffer solution, $\mathrm{pH}$ 7.0 , and subtracting the background oxidation charge. 
charges obtained for six different PPy-Urs-SCD polymer films are very similar, giving very good reproducibility.

Given its high selectivity and excellent sensing ability towards urea, the PPy-Urs-SCD film could potentially be used in the medical industry, particularly in the area of dialysis, to monitor urea concentrations in patients suffering from renal failure.

\section{Conclusions}

Although urease-containing polypyrrole films have been extensively studied, one of the major drawbacks is the sensitivity of these films towards urea. With this in mind, a novel urease-containing polypyrrole film, PPy-Urs-SCD, was successfully developed and characterised. The PPy-Urs-SCD film has a superior sensitivity of $5.79 \mu \mathrm{C} \mu \mathrm{M}^{-1}$ towards urea, compared to $0.76 \mu \mathrm{C} \mu \mathrm{M}^{-1}$ for the PPy-Urs-Cl polymer film. This can be accounted for by the sulfonated- $\beta$-cyclodextrin, SCD, which forms a 1:1 inclusion complex with urea, as established from the decrease in current with increasing SCD concentration, the lower diffusion coefficients for urea in the presence of SCD and the characteristic Job's plot with a maximum value at 0.5 . Because of its high sensitivity, this sensor may be useful in the detection of urea in the biomedical industry, particularly for patients suffering from renal disease. Future work will involve demonstrating the performance of the PPy-Urs-SCD film in the presence of other interfering compounds and investigating the detection limits of the film in real sample analysis.

\section{Acknowledgements}

The authors would like to thank the Irish Research Council for the financial support received for this project.

\section{References}

[1] M.-J. Syu, Y.-S. Chang, Biosens. Bioelectron. 24 (2009) 2671

[2] S. Cosnier, Biosens. Bioelectron. 14 (1999) 443.

[3] G. Maia, R.M. Torresi, E.A. Ticianelli, F.C. Nart, J. Phys. Chem. 100 (1996) 15910.

[4] A.L. Brisena, A. Baca, Q.Z. Zhou, R. Lai, F.M. Zhou, Anal. Chim. Acta 441 (2001) 123.

[5] E.M.M. Del Valle, Process Biochemistry 39 (2004) 1033

[6] G. Astray, C. Gonzalez-Barreiro, J.C. Mejuto, R. Rial-Otero, J. Simal-Gandara, Food Hydrocolloids 23 (2009) 1631.
[7] L.X. Song, L. Bai, X.M. Xu, J. He, S.Z. Pan, Coordination Chem. Rev. 253 (2009) 1276

[8] J. Szejtli, Trends in Biotechnology 7 (1989) 170.

[9] S. Hbaieb, R. Kalfat, Y. Chevalier, N. Amdouni, H. Parrot-Lopez, Mater. Sci. Eng. C. 28 (2008) 697.

[10] S.B. Adeloju, S.J. Shaw, G.G. Wallace, Anal. Chim. Acta 323 (1996) 107.

[11] T. Matsue, D.H. Evans, T. Osa, N. Kobayashi, J. Am. Chem. Soc. 107 (1985) 3411.

[12] Z.N. Gao, X.L. Wen, H.L. Li, Pol. J. Chem. 76 (2002) 1001

[13] A.F. Diaz, J.I. Castillo, J.A. Logan, W.Y. Lee, J. Electroanal. Chem. 129 (1981) 115

[14] J. Tietje-Girault, C. Ponce De Leon, F.C. Walsh, Surf. Coat. Technol. 201 (2007) 6025.

[15] C.E. Schmidt, J.M. Fonner, L. Forciniti, H. Nguyen, J.D. Byrne, Y.F. Kou, J. SyedaNawaz, Biomed. Mater. 3 (2008) 034124.

[16] A. Ramanavicius, A. Ramanaviciene, A. Malinauskas, Electrochimica Acta 51 (2006) 6025.

[17] T. de J. Licona-Sanchez, G.A. Alvarez-Romero, L.H. Mendoza-Huizar, C.A. GalanVidel, J. Phys. Chem. 114 (2010) 9737.

[18] T. de J. Licona-Sanchez, G.A. Alvarez-Romero, M. Palomar-Pardave, C.A. GalanVidel, M.E. Paez-Hernandez, M.T. Ramirez Silva, M. Romero-Romo, Int. J. Electrochem. Sci. 6 (2011) 1537.

[19] G. G. Wallace, G.M. Spinks, L.A. P. Kane-Maguire and P. R. Teasdale, Conductive Electroactive Polymers: Intelligent Materials Systems, CRC Press, 237, (2003).

[20] M. Singh, N. Verma, A.K. Garg, N. Redhu, Sens. Actuators B: Chem. 134 (2008) 345.

[21] G. Dhawan, G. Sumana, B.D. Malhotra, Biochem. Eng. J. 44 (2009) 42

[22] P. Bertocchi, D. Compagnone, G. Palleschi, Biosens. Bioelectron. 11 (1996) 1.

[23] H. Dodziuk, Cyclodextrins and their Complexes: Chemistry, Analytical Methods, Applications, Wiley-VCH, 489, (2006).

[24] M.S. Ibrahim, I.S. Shehatta, A.A. Al-Nayeli, J. Pharm. Biomed. Anal. 28 (2002) 217.

[25] X. Wang, H. Watanabe, N. Sekioka, H. Hamana, S. Uchiyama, Electroanalysis 19 (2007) 1300.

[26] P.M. Bersier, J. Bersier, B. Klingert, Electroanal. Chem. 3 (1991) 443.

[27] C. Yanez, L.J. Nunez-Vergara, J.A. Squella, Electroanal. Chem. 15 (2003) 1771

[28] Q.G. Nehring, J.W. Hightower, J.L. Anderson, J. Anal. Chem. 58 (1986) 2777.

[29] H. Demircigolu, H. Beyenal, A. Tanyolac, N. Hasirci, Polymer 23 (21) (1995) 4091.

[30] H. Miyama, T. Kobayashi, Y. Nosaka, Biotech. Bioeng. 24 (1982) 2757.

[31] B. Krajewska, M. Lezski, W. Zaborska, J. Chem. Tech. Biotech. 48 (1990) 337.

[32] A. Dimitrovska, B. Andonovski, K. Stojanoski, Int. J. Pharm. 134 (1996) 213.

[33] C.Y.Huang, R.X.Zhou, D.C.H. Yang, P.B. Chock, Biophysical Chemistry 100 (2003) 143.

[34] A. Sayago, M. Boccio, A.G. Asuero, Int. J. Pharm. 295 (2005) 29.

[35] G.-C. Zhao, J.-J. Zhu, J.-J. Zhang, H.-Y. Chen, Anal. Chim. Acta 394 (1999) 337.

[36] S. Letellier, B. Maupas, J.P. Gramond, F. Guyon, P. Gareil, Anal. Chim. Acta 315 (1995) 357.

[37] X.J. Dang, J. Tong, H.L. Li, J. Incl. Phenom. Macro. 24 (1996) 275.

[38] J. Szejtli, Chemical Reviews 98 (1998) 1743.

[39] A. Amini, T. Rundlof, M.B.G. Rydberg, T. Arvidsson, J. Sep. Sci. 27 (2004) 1102

[40] H. Zhao, Y. Zhang, Z. Yuan, Anal. Chim. Acta 441 (2001) 117.

[41] S. Suematsu, Y. Oura, H. Tsujimoto, H. Kanno, K. Naoi, Electrochim. Acta 45 (2000) 3813.

[42] P.C. Pandey, G. Singh, Talanta 55 (2001) 773. 